\title{
Difficulty in elaboration of coping strategy in foreign mother
}

\begin{abstract}
Culture and communication difficulties are barriers towards building a strong system of self-help for foreign mother of child affected by severe disease as leukemia, related to the lack of family support and difficulties in relationship. We report a short story about difficulty in elaboration of coping strategy in foreign mother.
\end{abstract}

Keywords: coping, cross-cultural care, cancer
Volume 2 Issue 3 - 2015

\author{
Daniela Caprino, Luisa M Massimo \\ Department of Pediatric Hematology and Oncology, G. Gaslini \\ Scientific Children's Hospital, Genova, Italy
Correspondence: Daniela Caprino, Department of Pediatric Hematology and Oncology, G. Gaslini Research Children's Hospital, Largo Gerolamo Gaslini 5, I6I47 Genova, Italy, Tel 39. 0I0-5636665, Fax 39-010-56367|4,.
Email danielacaprino@ospedale.gaslini.ge.it

Received: November 06, 2014 | Published: March II, 2015

\section{Introduction}

With the great migrations of people of any age the world has changed in the last decades. We can see it while we are walking along the street, while queuing at the post office, while taking our children to school, and when we are admitted to hospital. The change in the cultural environment has put foreigners in a dependent condition, including social adaptation, which is often exacerbated by discrimination. ${ }^{1}$ When a child succumbs to a sudden and severe illness, troubles may ensue primarily because the family's native ideologies, customs and ways of managing illness differ from those in their adopted country. According to data from national and local studies, mortality increases linearly with social disadvantages for a wide range of indicators at both an individual and a geographic level., ${ }^{2,3}$ Poor socio-economic conditions may impact heavily on health status in broader classes of immigrants. Indeed, access to appropriate health information may be impeded mainly by factors related to different languages and cultures.

Poor socio-economic conditions may impact heavily on health status in broader classes of immigrants. Indeed, access to appropriate health information may be impeded mainly by factors related to different languages and cultures. In most cases, new immigrants face the difficulty of adjusting to their new-found homes, including having to cope with the problems that led to the migration from their native countries in the first place, as well as trying to overcome the cultural and linguistic barriers, all that while keeping in mind problems of discrimination. Living in a foreign country mainly affects children, women and individuals with disabilities, resulting in reduced psychological and social well-being. ${ }^{4,5}$

A severe disease in childhood has a deep impact on interfamilial relationships, and is the major component of an evolving process. From diagnosis onwards, any element interfering with physical, cognitive, emotional or behavioral development should be avoided. The family acts as a disease-related stress container, bearing the major burden of the child's psychological adjustment. Living with disease requires the activation of psychological defense mechanisms, cognitive functions, perception, memory, communication, judgment, acceptance and emotions which taken together mean coping. The successful evolution of the coping process enables good quality of life and adaptation.
Parents and especially mothers are reported as being the main source of support their sick child. ${ }^{6,7}$ Parents are considered the main source of information regarding the disease. On several occasions parents are asked to carry out various aspects of disease management and therapy at home, including drug administration, catheter upkeep, mouth washes to prevent mucositis, and monitoring the child's reactions to therapy. This involvement may be perceived as a great responsibility and the mothers may feel alone because their partners do not support them. ${ }^{8}$

A great deal of immigrant mother of very sick children treated in our country are unable to elaborate an effective coping strategy, the different culture and communication difficulties are barriers towards building a strong system of self help. Here is a short dialogue with an Islamic mother.

Why are you crying? My husband has beaten me, my son is very sick, I feel helpless and alone. Please tell me what happened. I was with other mothers and the doctor came to talk with each one of us about our children. He is a man and while speaking he looked at me, in my eyes, as he does with everyone. My husband was furious. I feel angry because in our country children with such diseases are considered lost, because treatment in private centers is very expensive and we cannot afford it, we are a poor family, because we were compelled to come here with the help of a Parents' Association, because for the past year we have been living in their community home together with other people, sick children with their parents, because it is difficult for us to cohabit with them, because it is hard to communicate with anyone, and they do not feel our problems. I have four more children at home. Nobody understands me. I am angry at my destiny, I am angry because nobody can do anything to change things. My husband is also angry, he feels hopeless and I am his victim every day. Yes, I understand you. You know that your child has leukemia, but that it is not high risk that he will recover, that you will go back home with him. It will be hard, of course, to live here for several more months. You must also see that your son is a happy boy, he is playing, he is learning our language, he can communicate with other children and with our play workers and he is collaborative with the health care staff. Nobody can help me, because the anger is within me. My husband is not working, he is very unhappy but he will never leave me alone here, it is not our way. 


\section{Conclusion}

A diagnosis of cancer in a child is an event that has a strong impact on his/her family. Communication between health providers and patient/family binomial is an important aspect both in coping with the disease and in resilience. The insight linked to the decision of leaving one's home, the frustrating experience with the local health system that was not able to cure the child, and the feeling of loneliness must all be taken into consideration for a good therapeutic alliance.

On the other hand physicians and health providers face every day the challenge to cure patients with different cultures and languages. Never the less patient's compliance with the medical recommendation is closely related to a good communication and relationship that could be difficult in some instance.

As in this case differences in culturally behavior may cause discomfort, the disruption of the therapeutic alliance and anger. ${ }^{9}$ Anger; this is quite a common emotion among many immigrant parents of sick children with high risk diseases who are treated in our hospital for long periods of time, as pediatric oncology requires. This feeling affects their personal and social wellbeing. ${ }^{10}$ Self-blame is a common expression of their condition, and they are unable to make helpful self-sacrifices. They feel the emotion of anger as a result of what has happened to them, and they do not have the ability they need to activate a good defense mechanism, this mother feels as she is trapped by her child's disease and she experiences a loss in her self-monitoring capacity to build a coping strategy. ${ }^{11,12}$ Health care professionals may promote dialogue and encourage mothers to express their feelings and establish a mutual support in dealing with the child's illness. In some situation it must be take in to account that mothers may never have experienced to be involved in decisionmaking process that is automatically granted to both parents in Occidental countries.

This story suggests that may be useful to ask patients about their believes and their needs considering that it is difficult for health providers to know the aspects of the myriad of cultures, as well as every patient's situation is unique depending on both culture of origin and personal experiences. Understand cultural beliefs about childhood cancer and coping strategies, it allows implementing culturally sensitive health care approach.

Moreover the availability of interpreters should be improved. They can provide information about family cultural background and considering the complexity of medical communication, they can help to fill the gap between different cultures. Educational training in Global Health for young physicians and health workers, should give to new generations more knowledge and experience to care for patients in our globalized world.

\section{Acknowledgments}

None.

\section{Conflicts of interest}

Author declares there are no conflicts of interest.

\section{Funding}

None.

\section{References}

1. Mullan F. Immigration Pediatrics. Health Aff (Millwood). 2005;24(6):1619-1623.

2. Senior K. Health Migration and Childhood Cancer. Lancet Oncol. 2006;7(11):889.

3. Bhatia S. Disparities in Cancer Outcomes: Lessons Learned from Children with Cancer. Pediatr Blood Cancer. 2011;56(6):994-1002.

4. American Psychological Association. The mental health needs of immigrants.

5. Mendoza FS. Health Disparities and Children in Immigrant Families: A Research Agenda. Pediatrics. 2009;124(3):187-195.

6. Fletcher PC. My Child has Cancer: The Costs of Mother's Experiences of Having a Child with Pediatric cancer. Issues Compr Pediatr Nurs 2010;33(3):164-184.

7. Fletcher PC, Schneider MA, Harry RJ. How Do I Cope? Factors Affecting Mothers' Abilities to Cope with Pediatric Cancer. $J$ Pediatr Oncol Nurs. 2010;27(5):285-298.

8. da Silva FM, Jacob E, Nascimento LC. Impact of Childhood Cancer on Parents' Relationships: An Integrative Review. J Nurs Scholarsh. 2010;42(3):250-261.

9. Carrillo JE, Green AR, Betancourt JR. Cross-Cultural Primary Care: A Patient- Based Approach. Ann Intern Med. 1999;130(10):829-834.

10. Habermas T, Diel V. The Emotional Impact of Loss Narratives: Event Severity and Narrative Perspectives. Emotion. 2010;10(3):312-323.

11. Miedema B, Hamilton R, Fortin P, et al. You Can Only Take So Much, and It Took Everything Out of Me: Coping Strategies Used by Parents of Children with Cancer. Palliat Support Care . 2010;8(2):197-206.

12. Grootenhuis MA, Last BF. Adjustment and Coping by Parents of Children with Cancer: A Review of the Literature. Support Care Cancer. 1997; 5(6):466-484 